\title{
Gute Mädchen - Böse Jungen? Die Bedeutung von Mo- ral für die Erklärung von Geschlechterunterschieden bei delinquentem Verhalten
}

\author{
Bettina Doering
}

\section{Theoretischer Hintergrund}

Einer der bedeutsamsten Diskurse innerhalb der moralpsychologischen Forschung beschäftigt sich mit Geschlechterunterschieden hinsichtlich der Fähigkeit, moralische Urteile zu treffen. Den Impuls für diese Debatte gab die Beobachtung von Gilligan (u. a. Kohlberg \& Gilligan, 1971), wonach innerhalb des Stufenmodells der moralischen Urteilsfähigkeit von Kohlberg Mädchen im Vergleich zu Jungen häufiger auf einer niedrigeren Stufe des moralischen Urteilsvermögens eingeordnet wurden. Bei Kohlberg $(1974,1996)$ besteht das moralische Urteilsvermögen im Wesentlichen darin, moralische Dilemmata zu lösen. Diese können auf Basis mehr oder weniger gut elaborierter Urteile entschieden werden. Dabei handelt es sich insbesondere um Fragen der Gerechtigkeit und weniger um Fragen der Nächstenliebe, da Gerechtigkeit nach Verallgemeinerung und Unparteilichkeit verlangt. Diese Fähigkeit entwickelt sich nach Kohlberg innerhalb eines stufenförmigen Verlaufs von drei Urteilsebenen mit jeweils zwei Unterstufen (Kohlberg, 1974, 1996). Kohlberg ging davon aus, dass alle Menschen innerhalb ihrer kognitiven Entwicklung diese Stufen durchlaufen, wobei er keineswegs davon ausging, dass jeder Mensch sich bis zum höchsten (d. h. postkonventionellen) Niveau entwickeln würde. Im Gegenteil postulierte Kohlberg, dass nur sehr wenige Menschen die höchsten Stufen überhaupt erreichen würden. Problematisch an diesem Stufenmodell erschien Gilligan (Kohlberg \& Gilligan, 1971), dass Mädchen im Vergleich zu Jungen bei Untersuchungen häufiger auf Stufe drei eingeordnet wurden, wobei Jungen eher Stufe vier erreichten. Stufe drei und Stufe vier sind Unterstufen des konventionellen Niveaus, wobei auf Stufe drei nahe soziale Beziehungen die relevante Bezugsnorm für moralische Entscheidungen bilden. Auf Stufe vier hingegen entsteht die Einsicht, dass allgemein anerkannte Regeln notwendig sind um ein gesellschaftliches Zusammenleben zu ermöglichen. Dementsprechend besitzen Mädchen ein geringeres moralisches Urteilsvermögen als Jungen. Diese 
Feststellung war der Anlass für Gilligan (1984), die These der zwei moralischen Orientierungen zu formulieren, wobei sie von einer eher männlichen Gerechtigkeitsorientierung und einer eher weiblichen Fürsorgeorientierung ausging. Nach Gilligan geht die Gerechtigkeitsorientierung mit einer eher männlichen, individualistischen und separierten Selbstkonzeption einher. Sie beansprucht eine unabhängige Objektivität und neigt $\mathrm{zu}$ abstrakten Regeln. Im Gegensatz dazu ist die Fürsorgeorientierung eher weiblich. Die Selbstkonstruktion bezieht sich auf andere und speist sich aus nahen sozialen Beziehungen. Innerhalb dieser moralischen Orientierung wird sich im Besonderen am Wohlergehen anderer orientiert und harmonische Beziehungen sind in den konkret auftretenden Situationen von Interesse (Walker, 2006, S.95). Gilligans These, dass es eine männliche Gerechtigkeitsmoral und eine weibliche Fürsorgemoral gibt, wurde in einer Vielzahl von Studien untersucht. Innerhalb einer Metaanalyse (Jaffee $\&$ Hyde, 2000) wurden 113 Studien und Dissertationen untersucht, um zu überprüfen, ob nicht nur theoretisch, sondern auch empirisch Unterschiede zwischen den Geschlechtern hinsichtlich der moralischen Orientierungen existieren. Dabei ergaben sich nur geringe Unterschiede zwischen Jungen und Mädchen, welche aber eine Präferenz von Mädchen für die Fürsorgeorientierung und bei Jungen für eine Gerechtigkeitsorientierung indizierten. In über 70\% aller Studien gab es allerdings keine Geschlechterunterschiede der moralischen Orientierungen.

Obwohl bezüglich der beiden moralischen Orientierungen nur geringe Unterschiede zwischen den beiden Geschlechtern gefunden wurden und somit die These, dass Jungen und Mädchen unterschiedliche moralische Denkweisen besitzen, weitestgehend widerlegt wurde, hatte die Diskussion weitreichende Implikationen für die moralpsychologische Entwicklungsforschung. Insbesondere die Aufwertung der Fürsorgedimension förderte die Erforschung moralischer Emotionen wie beispielsweise Empathie. Empathie bezeichnet eine affektive Reaktion, die auf dem Verständnis und der Sorge um den emotionalen Zustand eines anderen Menschen besteht. Diese affektive Reaktion ähnelt den Emotionen bzw. antizipierten Emotionen des Gegenübers (Eisenberg, Spinrad \& Morris, 2006, S.518). Die Fähigkeit, Empathie für andere Menschen zu empfinden, entsteht in rudimentärer Form bereits im zweiten Lebensjahr (Hoffman, 2000) und nimmt bis ins Jugendalter zu (Eisenberg et al., 2006). Diese Entwicklung wird vor allem durch die zunehmende Fähigkeit zur Rollen- und Perspektivübernahme gefördert. Im Verlauf ihrer Entwicklung werden Kinder und Jugendliche immer wieder mit Situationen konfrontiert, in denen sie lernen, die Emotionen anderer zu verstehen. Ebenso wirken die klassischen 
Erziehungs- und Sozialisationsinstanzen (z. B. Eltern und Schule) auf die Entwicklung sozial angepasster Reaktionsweisen ein (Eisenberg et al., 2006, S.521). Die Forschung zu Geschlechterunterschieden bei empathischen Reaktionen zeigt, dass Mädchen höhere Ausprägungen von Empathie besitzen (Eisenberg, Zhou \& Koller, 2001; Karniol, Gabay, Ochion \& Harari, 1998; Olweus \& Endresen, 1998). Diese Unterschiede gehen auch mit den bestehenden Geschlechterstereotypen einher, wonach Mädchen empathischer und prosozialer sind als Jungen. Dabei variieren die Ergebnisse der Untersuchungen aber mit der Erhebungsmethode. Selbstberichtsstudien zeigen größere Unterschiede zwischen Jungen und Mädchen im Vergleich zu Studien, in denen die Reaktionen schwerer durch bewusste Kontrolle beeinflussbar sind (Eisenberg et al., 2006). Dementsprechend ist ein nicht unbedeutender Anteil der bestehenden Geschlechterunterschiede auf sozial erwünschtes Antwortverhalten zurückzuführen (Eisenberg \& Lennon, 1983), mit dem Jungen und Mädchen versuchen, prototypisch ihren Geschlechterrollen zu entsprechen. Unabhängig von diesem Befund deuten aber die Effektstärken im Allgemeinen auf eine höhere Empathie bei Mädchen hin.

Neben Empathie wurden moralische Emotionen auch verwendet, um die Bindung an ein moralisches Urteil zu erfassen (Krettenauer \& Eichler, 2006; Malti \& Buchmann, 2010; Nunner-Winkler, 2007; Nunner-Winkler, Meyer-Nikele \& Wohlrab, 2006). Gehen moralische Urteile mit negativen oder positiven Emotionen einher, so besteht erst die Möglichkeit, dass moralische Urteile auch handlungsleitend werden. In diesem Zusammenhang kann von moralischer Motivation gesprochen werden, wobei diese im Besonderen am Wissen um die Verbindlichkeit des Gebotenen orientiert sein muss (Nunner-Winkler, 2008). „Moralische Motivation bedeutet die Bereitschaft des Handelnden, das, was er als richtig erkannt hat, auch unter persönlichen Kosten zu tun“ (Nunner-Winkler, 2008, S.103-104). Bei der Untersuchung von moralischer Motivation wurden bisher Vignetten mit moralischen Konflikten verwendet, wobei ein moralischer Wert und ein persönliches Bedürfnis miteinander in Konflikt stehen (Nunner-Winkler et al., 2006). Neben der Verhaltensentscheidung sind im Sinne Kohlbergs auch die Begründungen für das gewählte Verhalten relevant. Um die Bindung an das moralische Urteil zu erfassen, werden die Emotionen, die mit dem jeweiligen Urteil verbunden sind, abgefragt, wobei diese als Indikator für den Grad der Identifikation mit der gewählten Handlungsalternative verwendet werden. Treffen Personen beispielsweise eine unmoralische Entscheidung und antizipieren negative Emotionen nach der unmoralischen Handlung, wird diese Verhaltensalternative unwahrscheinlicher im 
Vergleich zu Personen, die annehmen sich auch danach noch gut zu fühlen. Insgesamt zeigt sich, dass Mädchen seltener als Jungen die unmoralische Handlungsalternative wählen. Dementsprechend besitzen Mädchen ebenso wie bei Empathie eine stärkere moralische Motivation als Jungen. Diese Unterschiede können zumindest teilweise auf eine starke Geschlechterrollenidentifikation zurückgeführt werden. Jungen mit einer stark ausgeprägten männlichen Rollenidentifikation besitzen die niedrigste moralische Motivation. Für Mädchen hat die Rollenidentifikationen keinen Einfluss (Nunner-Winkler et al., 2006). Die Autoren der Studie weisen aber darauf hin, dass die Ergebnisse vorsichtig interpretiert werden müssen, da die kausale Beziehung zwischen Geschlechterrollenorientierung und moralischer Motivation ungeklärt bleibt. Dass das Geschlecht und insbesondere die soziale Konstruktion des Geschlechts einen Einfluss auf die moralische Motivation haben könnte, wird auch durch einen weiteren Befund untermauert. Geschlechtsspezifische Rollenmuster spielen insbesondere in der Adoleszenz eine bedeutsame Rolle, so dass eine Zunahme der Geschlechterunterschiede hinsichtlich der moralischen Motivation im Jugendalter zu erwarten ist. Diese Annahme bestätigte sich bereits bei einem Vergleich unterschiedlicher Altersgruppen im Kindes- und Jugendalter (Doering, 2013; Nunner-Winkler, Meyer-Nikele \& Wohlrab, 2007).

Neben der Vernachlässigung von Emotionen innerhalb der Kohlbergtradition wurde u. a. von Walker und Pitts (1998, S.403) auf ein weiteres Problem von Kohlbergs Stufenmodell hingewiesen. Kohlberg (1981) ging davon aus, dass mit der Entwicklung zu einer höheren Stufe des moralischen Urteilsvermögens auch die Wahrscheinlichkeit ansteigt, sich diesen Einsichten entsprechend zu verhalten. Zwar gibt es einige Belege, wonach Personen höherer moralischer Urteilsstufe tatsächlich seltener delinquentes Verhalten zeigen (Stams et al., 2006). Dennoch trägt nach Lapsley (1996) nicht ausschließlich das Wissen um die Geltung eines moralischen Prinzips zu dessen Befolgung bei, sondern erst die Bedeutung dieses Prinzips für die eigene Selbstdefinition erhöht die moralische Motivation innerhalb einer konkreten Situation und somit die Wahrscheinlichkeit einer moralischen Handlung. Aus diesem Grund wurde im Besonderen von Blasi (1983) das moralische Selbst als bedeutsame Quelle moralischer Motivation eingeführt, wobei zwei Teildimensionen des moralischen Selbst unterschieden werden: Zum einen die moralische Selbstintegration und zum anderen die moralische Zentralität (Blasi, 1995, 2004, 2005). Moralische Selbstintegration bezieht sich vor allem auf die Frage in wie weit moralische Werte als internal oder external erlebt werden. Moralische Zentralität bezieht sich auf die Bedeutsamkeit moralischer Eigenschaften für das 
Selbstkonzept. Beispielsweise kann Fairness eine sehr wichtige moralische Eigenschaft für das Selbstkonzept sein. Diese Bedeutsamkeit kann aber als ausschließlich external, das heißt von der Gesellschaft oder den Eltern erwünscht wahrgenommen werden. Die Dimension der moralischen Zentralität hat in der empirischen Forschung im Anschluss an das ,Self Model“" von Blasi (1983) eine stärkere Rezeption genossen (Arnold, 1993; Barriga, Morrison, Liau \& Gibbs, 2001; Doering, 2013; Krettenauer, 2011; Nunner-Winkler et al., 2006). Lediglich Arnold (1993) untersuchte beide Dimensionen an Schülern der sechsten, achten, zehnten und zwölften Jahrgangsstufe und fand bei den jüngeren Teilnehmern der Studie eine eher externale Perspektive und mit zunehmenden Alter eine Stärkung der internalen Perspektive. Bezüglich moralischer Zentralität gab es keine entwicklungsbedingten Unterschiede zwischen den Altersgruppen. In Bezug auf die moralische Zentralität finden sich Befunde, wonach Mädchen eine stärkere Identifikation mit moralischen Eigenschaften aufweisen (Arnold, 1993; Doering, 2013).

Die dargestellten Ergebnisse zu Empathie, moralischer Motivation und dem moralischen Selbst zeigen, dass Mädchen im Vergleich zu Jungen eine stärker ausgeprägte Empathie bzw. eine höhere moralische Motivation aufweisen und auch moralischen Eigenschaften eine größere Bedeutung beimessen. Damit muss Gilligans (Gilligan, 1984; Nunner-Winkler, 1991) Kritik vor allem auf das Stufenmodell von Kohlberg und das moralische Urteilsvermögen begrenzt bleiben.

Da moralpsychologische Entwicklungsforschung immer auch die Erklärung von tatsächlichem Verhalten im Blick hat, sind die dargestellten Geschlechterunterschiede bei Empathie, moralischer Motivation und der Zentralität auch für die Erklärung delinquenten Verhaltens relevant. Der Zusammenhang zwischen Moral und delinquenten Verhalten ergibt sich u. a. daraus, dass delinquentes, abweichendes oder auch kriminelles Verhalten nicht nur einen rechtlichen oder konventionellen, sondern insbesondere bei schweren Deliktsformen auch einen moralischen Regel- bzw. Normbruch darstellt. Dass Moral einen bedeutsamen Einfluss auf delinquentes Verhalten besitzt, wurde bereits in verschiedenen Studien nachgewiesen (Antonaccio \& Tittle, 2008; Murray-Close, Crick \& Galotti, 2006; Stams et al., 2006; Svensson, Pauwels \& Weerman, 2010; Wikström \& Svensson, 2010). Auch für die hier verwendeten Teildimensionen deuten die bestehenden Studien auf delinquenzreduzierende Einflüsse hin. Diesen Ergebnissen folgend, könnten Empathie, moralische Motivation und die Zentralität moralischer Eigenschaften auch die Unterschiede zwischen Jungen und Mädchen hinsichtlich delinquenten Verhaltens erklären. 
Studien berichten übereinstimmend, dass Straftaten häufiger von Männern als von Frauen begangen werden (Schneider, 2007). Zu diesem Ergebnis kommen Analysen mit Hellfeld- und Dunkelfeldstatistiken (Baier, 2011). Die bestehenden Unterschiede variieren allerdings mit der Schwere des jeweiligen Deliktes. Beispielsweise werden indirekte, relationale oder soziale Aggressionsformen von Mädchen mindestens genauso häufig wie von Jungen begangen (Scheithauer, 2003). Schwere Straftaten wie beispielsweise Körperverletzungen, Raub und Einbruch werden von Jungen bzw. Männern hingegen erheblich häufiger begangen. Innerhalb einer repräsentativen, deutschlandweiten Befragung von fast 45,000 Jugendlichen (9. Jahrgangsstufe) berichten Jungen mit $17.8 \%$ etwa dreimal so häufig wie Mädchen, eine Körperverletzung begangen zu haben. Bei der Betrachtung der Gewaltkriminalität, d. h. leichte bzw. schwere Körperverletzung, Raub, Erpressung und sexuelle Gewalt liegen die Werte etwa viermal höher (9.0 vs. $2.1 \%$ ). Leichtere Deliktsformen unterscheiden sich zwischen Jungen und Mädchen in einem geringeren Ausmaß. Ladendiebstähle werden beispielsweise von etwa genauso vielen Mädchen (12.4\%) wie Jungen (14.2\%) begangen. Auch in der polizeilichen Kriminalstatistik kann der Befund, wonach bei gewalttätigen bzw. schweren Straftaten stärkere Geschlechterunterschiede bestehen, bestätigt werden (Baier, 2011; Baier, Pfeiffer, Simonson \& Rabold, 2009).

Um zu überprüfen, ob Empathie, moralische Motivation und die moralische Zentralität die Unterschiede zwischen Jungen und Mädchen insbesondere hinsichtlich schweren delinquenten Verhaltens erklären kann, wird im Folgenden zunächst überprüft, ob substantielle Geschlechterunterschiede bezüglich der drei vorgestellten Teildimensionen von Moral bestehen. Es wird davon ausgegangen, dass Mädchen im Vergleich zu Jungen höhere Werte für Empathie, moralische Motivation und die Zentralität moralischer Eigenschaften aufweisen (Hypothese 1). Im nächsten Schritt wird überprüft werden, ob Empathie, moralische Motivation und die Zentralität moralischer Eigenschaften schweres delinquentes Verhalten bei Mädchen und Jungen gleichermaßen erklären kann. Es werden keine Geschlechterunterschiede erwartet (Hypothese 2). Im letzten Schritt soll überprüft werden, ob die drei dargestellten Dimensionen der Moral einen Beitrag zur Erklärung der bestehenden Geschlechterunterschiede zwischen Jungen und Mädchen leisten können. Es wird davon ausgegangen, dass Empathie, moralische Zentralität und moralische Motivation einen Teil der bestehenden Geschlechterunterschiede des delinquenten Verhaltens erklären können (Hypothese 3). 


\section{Methode}

\subsection{Stichprobe}

Eine repräsentative, standardisierte und testleiteradministrierte Schülerbefragung innerhalb der siebten und neunten Jahrgangsstufe im Landkreis Emsland dient als Grundlage für die Untersuchung der formulierten Hypothesen. Ausschließlich Schüler, deren Eltern ihr Einverständnis für die Teilnahme an der Studie erklärten, nahmen an der Studie teil. Die Rücklaufquote liegt in der 7. Klasse bei $74.9 \%$ und in der 9. Klasse bei $72.0 \%$. Insgesamt nahmen in der 7. Klasse 815 Schüler und in der 9. Klasse 2,891 Schüler an der Befragung teil. Da keine altersspezifischen Annahmen für die hier formulierten Forschungsfragen gemacht wurden, werden die beiden Jahrgänge im Folgenden gemeinsam analysiert. Der Stichprobenumfang beträgt 3,706 Personen, wobei die Schüler im Durchschnitt 14.76 Jahre $(\mathrm{SD}=1.01)$ sind. $49.0 \%$ der Schüler sind männlich. Der überwiegende Anteil der Jugendlichen besitzt keinen Migrationshintergrund $(83.7 \%)^{1}$.

\subsection{Messinstrumente}

\section{Empathie}

Empathie wurde mit einer verkürzten Version des Inventar zur Erfassung von Impulsivität, Risikoverhalten und Empathie bei 9- bis 14-jährigen Kindern erhoben (Stadler, Janke \& Schmeck, 2004). Für die Analysen wurden die vier trennschärfsten Items $\left(r_{\text {it }}>.60\right)$ der Subskala Empathie verwendet (Mößle, 2012). Die Kinder und Jugendlichen wurden gebeten auf einer vierstufigen Likert-Skala von „,1-stimmt überhaupt nicht“ bis ,4stimmt völlig" anzugeben, in wie weit sie den Items zustimmen bzw. diese ablehnen (z. B. „Es bedrückt mich, wenn ich sehe, dass jemand ausgelacht wird“). Mittels Cronbach's $\alpha$ wurde die Reliabilität berechnet, die als gut beurteilt werden kann $(\alpha=.83)$.

1 Einen Migrationshintergrund besitzen die befragten Jugendlichen dann, wenn sie selbst oder ihre Eltern (d. h. Mutter oder Vater) eine nichtdeutsche Staatsangehörigkeit besitzen oder ein nichtdeutsches Geburtsland aufweisen. 


\section{Moralische Motivation}

Die hier verwendete Skala „Moralische Motivation“ versucht, auf „Basis hypothetischer Handlungsentscheidungen und Emotionszuschreibungen in moralischen Konflikten" die Stärke der moralischen Motivation eines Individuums abzubilden (Nunner-Winkler et al., 2006, S.66). In Anlehnung an Malti und Buchmann (2010) wurden aus den bei Nunner-Winkler et al. (2006) verwendeten fünf moralischen Konflikten zwei ausgewählt. Bei beiden Konflikten handelt es sich um validierte Erhebungsinstrumente (Nunner-Winkler et al., 2006).

Für den ersten Konflikt (Fahrradkonflikt) wurde den Studienteilnehmern folgender Wortlaut vorgelegt: „Stell dir vor, du möchtest dein Fahrrad verkaufen und dafür 400 Euro haben. Ein Schüler interessiert sich für das Fahrrad und handelt den Preis mit dir auf 320 Euro herunter. Er erklärt: „Ich habe im Moment leider kein Geld bei mir. Ich gehe schnell nach Hause und bin in einer halben Stunde wieder da.“ Du sagst: „Abgemacht. Ich warte auf dich." Kaum ist er weg, kommt ein anderer Kunde und bietet dir den vollen Preis, 400 Euro, für das Fahrrad." Bei dem zweiten Konflikt (Geldkonflikt) wurde folgende Formulierung gewählt: „Stell dir vor, du bist gerade auf dem Heimweg. Plötzlich siehst du einen Geldbeutel vor dir auf dem Gehweg liegen. In dem Geldbeutel findest du den Ausweis des Besitzers und 100 Euro."

Im Anschluss an die Darbietung der moralischen Konflikte wurden die Teilnehmer gebeten, anzugeben, was sie selbst in der vorgegebenen Situation tun würden, wie sie ihre Handlungsentscheidung begründen, welche Emotionen sie dabei hätten und warum sie diese Emotionen haben würden. Das offene Antwortformat wurde gewählt, da sogenannte ,production-measures“ im Vergleich zu „recognition-measures“ das Ausmaß sozial erwünschter Antworten reduzieren können (Elm \& Weber, 1994).

In Anlehnung an die von Nunner-Winkler et al. (2006) und Malti und Buchmann (2010) vorgestellten Auswertungsstrategien wurden die offenen Antworten der Jugendlichen kodiert und ausgewertet. Da die moralischen Konflikte zum ersten Mal nicht in einem mündlichen Interview, sondern in einer schriftlichen Befragung eingesetzt wurden, wurde ein Manipulationscheck verwendet, mit dem überprüft werden konnte, ob die Jugendlichen die Konflikte gelesen und verstanden hatten. Innerhalb des Fahrradkonfliktes antworteten $3.2 \%$ der Siebtklässler und $2.2 \%$ der Neuntklässler falsch auf die Frage worum es in der Vignette ging. Dies galt innerhalb des Geldkonfliktes für 1.9\% der Siebtklässler und lediglich für $0.8 \%$ der Neuntklässler. 
Die erhobenen Daten wurden von zwei geschulten studentischen Hilfskräften kodiert. Die Kodierungen wurden mittels eines induktiv abgeleiteten Kategoriensystems vorgenommen. Das Kategoriensystem und dessen Reliabilität wurde vor der Anwendung an 9.5\% der Fälle überprüft. Die Interraterreliabilität (Cohen's Kappa) lag für alle Kategorien über .70, was in gängigen Klassifikationen als gute Übereinstimmung bezeichnet wird (Bortz \& Döring, 2005, S.277).

Für die folgenden Analysen wurde ein Globalrating gebildet, welches letztendlich drei Ausprägungen besitzt. Die erste Kategorie (niedrige moralische Motivation) umfasst all diejenigen Jugendlichen, die sich in beiden Konflikten für die eher ,unmoralische“ Handlungsalternative entscheiden, das heißt die größere Summe des Geldes im Fahrradkonflikt bevorzugen bzw. den Geldbeutel nicht an den Besitzer zurückbringen. Die mittlere Kategorie (mittlere moralische Motivation) umfasst alle Personen, die in einem der beiden Konflikte die unmoralische Alternative wählen. Die letzte Kategorie (hohe moralische Motivation) bezieht sich auf Personen, die in beiden moralischen Konflikten die moralische Handlungsalternative wählen, das heißt im Fahrradkonflikt die Abmachung einhalten und auf den ersten Kunden warten bzw. im Geldkonflikt versuchen, dem Besitzer das Portemonnaie mit dem vollständigen Inhalt aushändigen wollen. Alle Personen, die im Folgenden in die Analyse eingehen, haben bei mindestens einem der beiden Konflikte bei der Entscheidung, den Begründungen und Emotionen vollständige Werte. Diejenigen mit lediglich einem vollständig beantworteten Konflikt wurden je nach moralischer bzw. unmoralischer Entscheidung in die erste bzw. letzte Kategorie eingeordnet, da bei ihnen keine widersprüchlichen Konfliktlösungen vorlagen. Insgesamt wurde der Fahrradkonflikt von 13.5\% $(\mathrm{N}$ fehlend $=501)$ der Schüler nicht beantwortet, der Geldkonflikt von 7.2\% $(\mathrm{N}$ fehlend $=265)$ der Schüler. Auf beiden Konflikten keinen gültigen Wert besitzen 9.1\% $(\mathrm{N}$ fehlend = 337) Fälle.

\section{Moralische Zentralität}

Die Zentralität moralischer Eigenschaften wurde mittels einer adaptierten Version des sogenannten „Good Self-Assessment“ von Barriga et al. (2001) erhoben. Ähnlich strukturierte Varianten dieses Messinstrumentes finden sich in verschiedenen weiteren Publikationen zum moralischen Selbst (Arnold, 1993; Harter \& Monsour, 1992; Nunner-Winkler et al., 2007; Pratt, Hunsberger, Pancer \& Alisat, 2003; Pratt, Arnold, Pratt \& 
Diessner, 1999). Aus den bestehenden Publikationen wurden insgesamt vier moralische Eigenschaften entnommen, die zum einen für beide untersuchten Altersgruppen bedeutsam sind und eindeutig moralische Eigenschaften ausweisen (Doering, 2013). Die Jugendlichen wurden gebeten, auf einer vierstufigen Likert-Skala von „1-nicht wichtig“ bis ,4-sehr wichtig“" anzugeben, wie bedeutsam die Eigenschaften fair, ehrlich, hilfsbereit und rücksichtsvoll für ihre Selbstdefinition sind. Die interne Konsistenz liegt in einem befriedigenden Bereich $(\alpha=.71)$.

\section{Delinquentes Verhalten}

Delinquentes Verhalten wurde mit zwei verschiedenen Delikttypen operationalisiert: schwerer Diebstahl und Gewaltdelikte. Unter schwerem Diebstahl wird Einbruch und Fahrzeugdiebstahl gefasst. Gewaltdelikte umfassen leichte und schwere Körperverletzung, Raub, Erpressung und sexuelle Gewalt (Doering \& Baier, 2011). Der Referenzzeitraum beträgt 12 Monate. Die einzelnen Delikte wurden für die Kategorie schwerer Diebstahl und Gewaltdelikte mittels eines „Maximalbefehls“ zusammengefasst, d. h. sobald eines der genannten Delikte begangen wurde ist eine Person Täter eines schweren Diebstahls bzw. eines Gewaltdeliktes. Es wird nicht unterschieden wie häufig ein Delikt begangen wurde bzw. ob mehrere verschiedene Delikte begangen wurden.

\section{Ergebnisse}

Bevor die drei zentralen Forschungsfragen überprüft werden sollen, erscheint es bedeutsam auch empirisch die korrelativen Beziehungen zwischen den drei gemessenen Dimensionen von Moral zu untersuchen. Die korrelativen Zusammenhänge sind in Tabelle 1 abgebildet und wurden mittels Sperman's Rho berechnet. Neben den Gesamtzusammenhängen werden die Korrelationen auch getrennt für die beiden Geschlechter angegeben. Empathie korreliert in einem mittleren Bereich mit moralischer Zentralität. Dies gilt auch für die getrennten Analysen für Jungen und Mädchen. Moralische Motivation hingegen korreliert auf einem niedrigen Niveau mit Empathie und moralischer Zentralität, wobei die Korrelationen für Mädchen geringer ausfallen. Eine mögliche Ursache für die geringeren Zusammenhänge mit moralischer Motivation ist die Messung dieses Konstrukts: Zum einen wurden qualitative Antwortformate verwendet, zum 
anderen erheben die verwendeten moralischen Konflikte konkrete Situationen, wohingegen Empathie und die Zentralität moralischer Eigenschaften über ein globaleres Maß erfasst wurden.

Gleichwohl zeigt die Zusammenhangsanalyse, dass nicht nur die theoretische Trennung dieser drei Dimensionen sinnvoll ist, sondern dass alle drei Konstrukte unterschiedliche Facetten besitzen, die von keiner der anderen Dimensionen abgebildet werden kann.

Tabelle 1. Korrelationen zwischen den drei Dimensionen von Moral.

\begin{tabular}{|c|c|c|c|}
\hline & & Empathie & Moralische Motivation \\
\hline \multirow[t]{3}{*}{ Empathie } & Gesamt & & $.24 * * *$ \\
\hline & Jungen & & $.22 * * *$ \\
\hline & Mädchen & & $.15^{* * *}$ \\
\hline \multirow{3}{*}{$\begin{array}{l}\text { Moralische } \\
\text { Zentralität }\end{array}$} & Gesamt & $.42 * * *$ & $.22 * * *$ \\
\hline & Jungen & $.36^{* * *}$ & $.22 * * *$ \\
\hline & Mädchen & $.41 * * *$ & $.10 * * *$ \\
\hline
\end{tabular}

Anmerkung. *** $p<.001$.

Die erste Hypothese beschäftigt sich mit den Geschlechterunterschieden bezüglich der Ausprägungen von Empathie, der moralischen Motivation und der Zentralität moralischer Eigenschaften für das Selbstkonzept. Es wird angenommen, dass sich für alle drei Konzepte höhere Ausprägungen für Mädchen im Vergleich zu Jungen ergeben. Da es sich bei Empathie und der Zentralität moralischer Eigenschaften um intervallskalierte Variablen handelt, können t-Tests für unabhängige Stichproben mit Geschlecht als unabhängiger und Empathie bzw. der Zentralität moralischer Eigenschaften als abhängigen Variablen berechnet werden. Bei der moralischen Motivation liegt eine Ordinalskalierung vor, so dass für die Untersuchung des Zusammenhangs mit dem Geschlecht ein $\chi^{2}$-basiertes Verfahren namentlich Cramer's V verwendet wird.

Die Ergebnisse der t-Tests sind in Tabelle 2 dargestellt, wobei deutlich wird, dass Jungen signifikant niedrigere Werte hinsichtlich der Empathie und der Zentralität moralischer Eigenschaften aufweisen. Die mittlere Differenz ist dabei für Empathie etwas größer als dies für die moralische Zentralität der Fall ist. 
Tabelle 2. Empathie und moralische Zentralität im Geschlechtervergleich (Mittelwerte/ Standardabweichungen).

\begin{tabular}{|c|c|c|c|c|}
\hline & & M (SD) & t-Wert & Signifikanz \\
\hline Empathie & $\begin{array}{l}\text { Jungen } \\
\text { Mädchen }\end{array}$ & $\begin{array}{l}2.68(0.66) \\
3.20(0.52)\end{array}$ & -26.03 & $p<.01$ \\
\hline $\begin{array}{l}\text { Zentralität morali- } \\
\text { scher Eigenschaften }\end{array}$ & $\begin{array}{l}\text { Jungen } \\
\text { Mädchen }\end{array}$ & $\begin{array}{l}3.39(0.46) \\
3.56(0.38)\end{array}$ & -11.92 & $p<.01$ \\
\hline
\end{tabular}

Im nächsten Schritt soll mittels $\chi^{2}$-Test der Vergleich zwischen Jungen und Mädchen bezüglich der moralischen Motivation durchgeführt werden. In Abbildung 1 wird sichtbar, dass mehr Jungen als Mädchen eine niedrige moralische Motivation (16.7 vs. 3.2\%) besitzen, wohingegen sich das Verhältnis bei hoher moralischer Motivation (39.6 vs. 59.5\%) umkehrt. Der Anteil der Mädchen liegt etwa 20\% über dem der Jungen. Eine mittlere moralische Motivation besitzen etwas mehr Jungen als Mädchen (43.7 vs. 37.3\%). Die Berechnung von Cramer's V ergab signifikante Unterschiede zwischen Jungen und Mädchen bei der moralischen Motivation (Cramer's V $=.26, p<.01$ ).

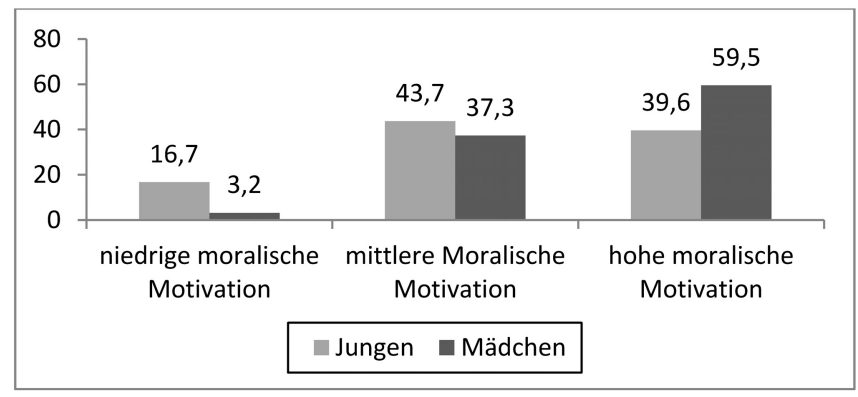

Abbildung 1. Moralische Motivation im Geschlechtervergleich (in \%).

Mittels der zweiten Forschungsfrage soll geklärt werden, ob der Zusammenhang zwischen den Dimensionen der Moral und delinquentem Verhalten für Jungen und Mädchen gleichermaßen besteht. Um diese Annahmen zu überprüfen, werden logistische Regressionen getrennt für Jungen bzw. Mädchen und schweren Diebstahl bzw. Gewaltdelikte berechnet. Insgesamt haben 3.4\% aller Jugendlichen in den letzten 12 Monaten einen schweren Diebstahl verübt. Ein Gewaltdelikt wurde in den letzten 12 Monaten von $6.4 \%$ aller Jugendlichen begangen. Die Klassenstufe wird als Kontrollvariable in das Modell aufgenommen. 
Tabelle 3. Logistische Regressionen für schweren Diebstahl und Gewalt getrennt nach Geschlecht, Odds Ratios $\left(\operatorname{Exp}(\mathrm{B}), p<.05^{*}, p<.01^{* *}\right)$.

\begin{tabular}{lcccc}
\hline & \multicolumn{2}{c}{ Schwerer Diebstahl } & \multicolumn{2}{c}{ Gewalt } \\
& Jungen & Mädchen & Jungen & Mädchen \\
\hline $\begin{array}{l}\text { Klassenstufe } \\
(2=9 . \text { Klasse) }\end{array}$ & 1.777 & 4.694 & 1.063 & 2.863 \\
$\begin{array}{l}\text { Empathie } \\
\text { Moralische Moti- }\end{array}$ & 0.789 & 0.465 & $0.534^{* *}$ & 0.645 \\
vation (niedrig) & Ref. & Ref. & Ref. & Ref. \\
$\begin{array}{l}\text { Moralische Moti- } \\
\text { vation (mittel) }\end{array}$ & $0.413^{* *}$ & $0.190^{*}$ & $0.469^{* *}$ & $0.339^{*}$ \\
$\begin{array}{l}\text { Moralische Moti- } \\
\text { vation (hoch) }\end{array}$ & $0.156^{* *}$ & $0.200^{*}$ & $0.147^{* *}$ & $0.156^{* *}$ \\
$\begin{array}{l}\text { Moralische Zent- } \\
\text { ralität }\end{array}$ & 0.694 & 0.820 & & \\
$\mathbf{N}$ & & & 0.990 & $0.328^{*}$ \\
Nagelkerkes R & & & & \\
\hline
\end{tabular}

In Tabelle 3 sind die Odds Ratios abgebildet, wobei Werte kleiner eins angeben, dass die Wahrscheinlichkeit, einen schweren Ladendiebstahl bzw. ein Gewaltdelikt zu begehen, im Vergleich der Gruppen (z. B. 7. Klasse vs. 9. Klasse) reduziert ist. Werte über eins indizieren das Gegenteil. Es ist zunächst für schweren Diebstahl zu erkennen, dass lediglich die moralische Motivation signifikant reduzierende Effekte auf schwere Diebstahlsdelikte besitzt. Weder Empathie noch moralische Zentralität reduzieren bei Jungen und Mädchen, die Wahrscheinlichkeit einen schweren Diebstahl zu begehen. Im Hinblick auf die Gewaltdelikte liegen unterschiedliche Effekte vor. Zwar hat moralische Motivation auch bei diesem Deliktstyp signifikante Effekte bei beiden Geschlechtern. Moralische Zentralität ist aber lediglich bei den Mädchen signifikant; Empathie lediglich bei den Jungen.

Nach den dargestellten Analysen wird deutlich, dass zumindest auf den ersten Blick doch unterschiedliche Wirkungen der einzelnen Dimensionen von Moral für Jungen bzw. Mädchen bestehen könnten. Um dies überprüfen zu können wurden zwei logistische Regressionen mit den Interakti- 
onstermen aus Geschlecht und den Dimensionen von Moral berechnet ${ }^{2}$. Die Interaktionsvariablen geben Aufschluss darüber, ob tatsächlich die Stärke des Einflusses von Empathie, moralischer Motivation und moralischer Zentralität zwischen Jungen und Mädchen verschieden ist. Lediglich die Interaktion von moralischer Zentralität und Geschlecht ist auf dem 5\%-Niveau signifikant. Dies indiziert, dass moralische Zentralität einen bedeutsameren Einfluss bei der Erklärung von Gewalttäterschaft bei Mädchen besitzt. Bei Jungen hingegen ist moralische Zentralität nicht relevant.

Mit der dritten Forschungsfrage soll überprüft werden, in wie weit die erhobenen Dimensionen von Moral die Unterschiede im Hinblick auf delinquentes Verhalten zwischen Jungen und Mädchen erklären können. Dabei wird davon ausgegangen, dass Empathie, moralische Motivation und moralische Zentralität die bestehenden Unterschiede zwischen Jungen und Mädchen bei schwerem Ladendiebstahl und bei Gewaltdelikten erklären können. Um diese Annahme zu überprüfen wurden jeweils zwei Modelle für schweren Diebstahl bzw. für Gewaltdelikte berechnet. Im ersten Modell wurden lediglich die Klassenstufe als Kontrollvariable und das Geschlecht als unabhängige Variable aufgenommen. Im nächsten Schritt wurden dann die drei Moraldimensionen integriert. Wenn Empathie, moralische Motivation und moralische Zentralität den Effekt des Geschlechts zumindest teilweise erklären, sollte sich das Odds Ratio von Geschlecht reduzieren. In Tabelle 4 kann dieser Effekt beobachtet werden: Unter Einbeziehung von moralischer Zentralität, Empathie und moralischer Motivation sinkt das Odds Ratio bei beiden Deliktstypen, d. h. zumindest teilweise können die Geschlechterunterschiede bei schwerem Diebstahl und Gewaltdelikten durch die einbezogenen Variablen erklärt werden. Bei schwerem Diebstahl und bei Gewaltdelikten ist die moralische Motivation ein signifikanter Prädiktor, wobei bei Gewaltdelikten zusätzlich Empathie signifikant ist.

2 Als Kontrollvariable wurde abermals die Klassenstufe aufgenommen. Neben den Haupteffekten von Geschlecht, Empathie, moralischer Motivation und moralischer Zentralität wurden die Interaktionsterme aus Geschlecht und den drei Dimensionen von Moral in das Modell integriert. 
Tabelle 4. Schrittweise logistische Regressionen für schweren Diebstahl.

\begin{tabular}{|c|c|c|c|c|}
\hline & \multicolumn{2}{|c|}{ Schwerer Diebstahl } & \multicolumn{2}{|c|}{ Gewalt } \\
\hline & Modell 1 & Modell 2 & Modell 1 & Modell 2 \\
\hline Schritt 1 & & & & \\
\hline Klasse $(2=9$. Klasse $)$ & $2.041^{*}$ & $2.136^{*}$ & 1.326 & 1.325 \\
\hline $\begin{array}{l}\text { Geschlecht } \\
(1=\text { Mädchen })\end{array}$ & $0.249^{* *}$ & $0.455^{* *}$ & $0.213^{* *}$ & $0.435^{* *}$ \\
\hline Schritt 2 & & & & \\
\hline Empathie & & 0.727 & & $0.559^{* *}$ \\
\hline $\begin{array}{l}\text { Moralische Motivation } \\
\text { (niedrig) }\end{array}$ & & Ref. & & Ref. \\
\hline $\begin{array}{l}\text { Moralische Motivation } \\
\text { (mittel) }\end{array}$ & & $0.361^{* *}$ & & $0.442^{* *}$ \\
\hline $\begin{array}{l}\text { Moralische Motivation } \\
\text { (hoch) }\end{array}$ & & $0.195^{* *}$ & & $0.159^{* *}$ \\
\hline Moralische Zentralität & & 0.699 & & 0.801 \\
\hline $\mathbf{N}$ & 2.526 & 2.526 & 2.524 & 2.524 \\
\hline Nagelkerkes $\mathbf{R}^{2}$ & 0.06 & 0.12 & 0.08 & 0.18 \\
\hline
\end{tabular}

Anmerkung. Odds Ratios $\left(\operatorname{Exp}(\mathrm{B}) .{ }^{*} p<.05,{ }^{* *} p<.05\right.$.

\section{Diskussion}

Im Fokus der dargestellten Analysen stand der Zusammenhang zwischen Moral, delinquentem Verhalten und Geschlecht. Ausgehend von der Debatte um eine geschlechterspezifische Moral (Gilligan, 1984; Kohlberg \& Gilligan, 1971) geht die erste Hypothese der Frage nach, ob überhaupt Geschlechterunterschiede bei den Konstrukten Empathie, moralische Motivation und moralische Zentralität bestehen. Alle drei Konstrukte werden als Teilkomponenten von Moral aufgefasst, wobei sie unterschiedliche Schnittmengen besitzen. Empathie bildet dabei die emotionale Komponente von Moral ab. Moralische Motivation versucht den leitenden Gedanken von Kohlberg (1974, 1996), wonach nicht ausschließlich die Entscheidungen, sondern die Begründungen relevant für die Beurteilung der moralischen Urteile sind aufzunehmen. Zusätzlich werden mittels Emotionszuschreibungen die kognitive und emotionale Komponente miteinander in Bezug gesetzt und auf konkrete, realitätsnahe Situationen bezogen (Nunner-Winkler, 2008; Nunner-Winkler et al., 2006). Moralische Zentralität bildet die persönlichkeitsbezogene Komponente von Moral und wird im Sinne der Eigenschaftstheorien (Scheffer \& Heckhausen, 2006) der Motivation als Quelle moralischer Motivation betrachtet (Doering, 
2013). Es zeigen sich zwischen allen drei Teilkomponenten geringe bis mittlere Zusammenhänge.

Anschließend an die Überprüfung der korrelativen Zusammenhänge wurde zunächst die erste Hypothese geprüft. Auf allen drei gemessenen moralischen Dimensionen, d. h. Empathie, moralischer Motivation und der moralischen Zentralität, besitzen Mädchen höhere Ausprägungen als Jungen, so dass Hypothese eins beibehalten werden kann. Diese Ergebnisse entsprechen bereits bestehenden Studien, die auch für Mädchen höhere Werte bei Empathie, moralischer Motivation und moralischer Zentralität nachweisen können (Arnold, 1993; Eisenberg \& Lennon, 1983; Karniol et al., 1998; Nunner-Winkler et al., 2006, 2007). Inwieweit die Ergebnisse durch ein sozial erwünschtes Antwortverhalten beeinflusst sind, kann die vorliegende Studie nicht beantworten. Mädchen könnten durchaus auch deshalb höhere Ausprägungen bei Empathie, moralischer Motivation und moralischer Zentralität aufweisen, da sie versuchen, einem eher ,weiblichen" prosozialen Geschlechterstereotyp zu entsprechen. Für Jungen könnten die Antworttendenzen eher durch ein „männliches, cooles“ Geschlechterbild geprägt sein, in welchem es durchaus vorkommt nicht immer rücksichtsvoll und hilfsbereit zu sein. Insbesondere die Studie von Nunner-Winkler et al. (2006) weist darauf hin, dass vor allem Jungen durch ihre Geschlechterrollenidentifikation beeinflusst sind und dem entsprechend eine geringere moralische Motivation berichten. Unabhängig von dieser Möglichkeit zeigen aber Studien, dass selbst unter Kontrolle möglicher Verzerrungen durch soziale Erwünschtheit, die Unterschiede zwischen Jungen und Mädchen zwar geringer sind, aber dennoch bestehen bleiben (Eisenberg et al., 2006).

Für die geschlechterspezifischen Zusammenhangsanalysen zwischen den Komponenten von Moral und delinquentem Verhalten besitzen die Verzerrungen durch sozial erwünschtes Antwortverhalten eine geringere Bedeutung. Für beide Delikte und Geschlechter besitzt moralische Motivation einen signifikanten Einfluss, wobei für die Gewaltdelikte bei Jungen Empathie und bei den Mädchen moralische Zentralität einen zusätzlichen Einfluss besitzt. Durch die Berechnung von Interaktionstermen konnte ermittelt werden, dass lediglich die moralische Zentralität einen geschlechterspezifischen Einfluss auf Gewaltdelikte besitzt. Moralische Zentralität senkt lediglich für die Mädchen die Wahrscheinlichkeit ein Gewaltdelikt zu begehen. Eine mögliche Erklärung wäre auch hier die Geschlechterrollenorientierung der Jungen. Moralische Werte könnten für Jungen hinter die klassisch männlichen Attribute bei der Identitätsbildung innerhalb der Adoleszenz zurückgestellt werden. Diese Erklärungsmög- 
lichkeit wäre anschlussfähig an die bereits diskutierten Ergebnisse von Nunner-Winkler et al. (2006, 2007).

Abschließend wurde die letzte der drei Hypothesen geprüft. Die Ergebnisse bestätigen die Hypothese, wonach die drei Teilkomponenten der Moral zumindest teilweise die Geschlechterunterschiede bei schwerem delinquenten Verhalten erklären können. Dabei kommt der moralischen Motivation der bedeutsamste Einfluss zu. Dies kann verschiedene Gründe haben. Einerseits weisen die beiden verwendeten Konflikte eine höhere Verhaltensnähe auf, so dass sie den weniger verhaltensnahen Messinstrumenten überlegen sein könnten. Andererseits ist es möglich, dass die konkreten Entscheidungen in den beiden moralischen Konflikten indikativer für die tatsächliche Moral der Jugendlichen sind als die globalen Skalen der Empathie und der moralischen Zentralität. Dass moralische Motivation für beide Deliktstypen, Eigentums- und Gewaltdelikte, gleichermaßen einen Teil der Geschlechterunterschiede erklären kann, unterstreicht ihre Bedeutung bei der Erklärung delinquenten Verhaltes.

$\mathrm{Da}$ aber nur ein Teil der bestehenden Geschlechterunterschiede bei schwerem kriminellen Verhalten durch Moral erklärt werden kann, sind weitere Erklärungsansätze bedeutsam. Neben der bereits genannten Geschlechterrollenorientierung werden von Scheithauer (2003) beispielsweise biologische, tiefenpsychologische bzw. triebtheoretische und kognitivistische und lerntheoretische Ansätze als Erklärungen für die Geschlechterunterschiede bei delinquentem Verhalten genannt. Dabei ist unklar welchen konkreten Anteil die Moralentwicklung im Vergleich zu weiteren Einflussfaktoren besitzt. Dem entsprechend sollte sich nachfolgende Forschung der Bedeutung moralischer Entwicklung im Vergleich zu weiteren möglichen Einflussfaktoren widmen.

\section{Literatur}

Antonaccio, O. \& Tittle, C. R. (2008). Morality, self control and crime. Criminology, 46, 479-509.

Arnold, M. L. (1993). The place of morality in the adolescent self. Harvard University, Cambridge.

Baier, D. (2011). Jugendgewalt und Geschlecht - Erkenntnisse aus Kriminalstatistik und Dunkelfelduntersuchungen. Zeitschrift für Jugendkriminalrecht und Jugendhilfe, 22, 356-364. 
Baier, D., Pfeiffer, C., Simonson, J. \& Rabold, S. (2009). Jugendliche in Deutschland als Opfer und Täter von Gewalt. Erster Forschungsbericht zum gemeinsamen Forschungsprojekt des Bundesministerium des Innern und des KFN (Forschungsbericht No. 107). Hannover: Kriminologisches Forschungsinstitut Niedersachsen.

Barriga, A. Q., Morrison, E. M., Liau, A. K. \& Gibbs, J. C. (2001). Moral cognition: Explaining the gender difference in antisocial behavior. Merrill-Palmer Quarterly, 47, 532-562.

Blasi, A. (1983). Moral cognition and moral action: A theoretical perspective. Developmental Review, 3, 178-210.

Blasi, A. (1995). Moral understanding and the moral personality: The process of moral integration. In W. M. Kurtines \& J. L. Gewirtz (Hrsg.), Moral development: An introduction (Bd. 1, S. 229-253). Boston: Allyn and Bacon.

Blasi, A. (2004). Moral functioning: Moral understanding and personality. In D. Lapsley \& D. Narvaez (Hrsg.), Moral Development, self, and identity (S. 335-348). Erlbaum: Mahwah, NJ.

Blasi, A. (2005). Moral character: A psychological approach. In D. Lapsley \& F. C. Power (Hrsg.), Character psychology and character education. (S. 67-100). Notre Dame: University of Notre Dame Press.

Bortz, J. \& Döring, N. (2005). Forschungsmethoden und Evaluation. Berlin: Springer.

Doering, B. (2013). The development of moral identity and moral motivation in childhood and adolescence. In K. Heinrichs, F. Oser \& T. Lovat (Hrsg.), Handbook of moral motivation (S. 289-307). Rotterdam: Sense Publishers.

Doering, B. \& Baier, D. (2011). Jugendliche als Opfer und Täter von Gewalt im Landkreis Emsland (Forschungsbericht No. 113). Hannover: Kriminologisches Forschungsinstitut Niedersachsen.

Eisenberg, N. \& Lennon, R. (1983). Gender differences in empathy and related capacities. Psychological Bulletin, 94, 100-131.

Eisenberg, N., Spinrad, T. L. \& Morris, A. (2006). Empathy-related responding in children. In M. Killen \& J. G. Smetana (Hrsg.), Handbook of moral development (S. 517-549). Mahwah: Lawrence Erlbaum Associates.

Eisenberg, N., Zhou, Q. \& Koller, S. (2001). Brazillian adolescents' prosocial moral judgment and behavior: Relations to sympathy, perspective taking, gender-role orientation, and demographic charac-teristics. Child Development, 72, 518-534.

Elm, D. R. \& Weber, J. (1994). Measuring moral judgement: The Moral Judgement Interview or the Defining Issue Test. Journal of Business Ethics, 13, 341-355.

Gilligan, C. (1984). Die andere Stimme. Lebenskonflikte und Moral der Frau. München: Piper.

Harter, S. \& Monsour, A. (1992). Developmental analysis of conflict caused by opposing attributes in the adolescent self-portrait. Developmental Psychology, 28, 251-260.

Hoffman, M. L. (2000). Empathy and moral development. Implications for caring and justice. Cambridge: Cambridge University. 
Jaffee, S. R. \& Hyde, J. S. (2000). Gender differences in moral orientation: A metaanalysis. Psychological Bulletin, 126(703-726).

Karniol, R., Gabay, R., Ochion, Y. \& Harari, Y. (1998). Is gender or gender-role orientation a better predictor of empathy in adolescence? Sex Roles, 39(45-59).

Kohlberg, L. (1974). Zur moralischen Entwicklung des Kindes: Drei Aufsätze. Frankfurt am Main: Suhrkamp.

Kohlberg, L. (1981). Essays on moral development I: The philosophy of moral development. Moral stages and the idea of justice. San Francisco: Harper \& Row.

Kohlberg, L. (1996). Die Psychologie der Moralentwicklung. Frankfurt: Suhrkamp.

Kohlberg, L. \& Gilligan, C. (1971). The adolescent as a philosopher: The discovery of the self in a post-conventional world. Daedalus, 100, 1051-1086.

Krettenauer, T. (2011). The dual moral self: Moral centrality and internal moral motivation. The Journal of Genetic Psychology, 172, 309-328.

Krettenauer, T. \& Eichler, D. (2006). Adolescents' self-attributed moral emotions following a moral transgression: Relations with delinquency, confidence in moral judgment and age. British Journal of Develop-mental Psychology, 24, 489-506.

Lapsley, D. K. (1996). Moral Psychology. Boulder: Westview.

Malti, T. \& Buchmann, M. (2010). Socialization and individual antecedents of adolescents' and young adults' moral motivation. Journal of Youth and Adolescence, 39, 138-149.

Mößle, T. (2012). dick, dumm, abhängig, gewalttätig? Problematische Mediennutzungsmuster und ihre Folgen im Kindesalter. Ergebnisse des Berliner Längsschnitt Medien. Baden-Baden: Nomos Verlag.

Murray-Close, D., Crick, N. R. \& Galotti, K. M. (2006). Children's moral reasoning regarding physical and relational aggression. Social De-velopment, 15, 345-372.

Nunner-Winkler, G. (1991). Weibliche Moral. Die Kontroverse um eine geschlechtsspezifische Ethik. Frankfurt am Main, New York: Campus.

Nunner-Winkler, G. (2007). Development of moral motivation from childhood to early adulthood. Journal of Moral Education, 36, 399-414.

Nunner-Winkler, G. (2008). Zur Entwicklung moralischer Motivation. In J. Schneider (Hrsg.), Entwicklung von der Kindheit bis zum Erwachsenenalter: Befunde der Münchner Längsschnittstudie LOGIK (S. 103-123). Weinheim: Beltz Verlag.

Nunner-Winkler, G., Meyer-Nikele, M. \& Wohlrab, D. (2006). Integration durch Moral: Moralische Motivation und Ziviltugenden Jugendlicher. Wiesbaden: VS Verlag für Sozialwissenschaften.

Nunner-Winkler, G., Meyer-Nikele, M. \& Wohlrab, D. (2007). Gender differences in moral motivation. Merrill-Palmer Quarterly, 53, 26-52.

Olweus, D. \& Endresen, I. M. (1998). The importance of sex-of-stimulus object: Age trends and sex differences in empathic responsiveness. Social Development, 7, 370388 .

Pratt, M. W., Arnold, M. L., Pratt, A. T. \& Diessner, R. (1999). Predicting adolescent moral reasoning from family climate: A longitudinal study. Journal of Early Adolescence, 19, 148-175. 
Pratt, M. W., Hunsberger, B., Pancer, S. M. \& Alisat, S. (2003). A longitudinal analysis of personal values socialization: Correlates of a moral self-ideal in late adolescence. Social Development, 12, 563-585.

Scheffer, D. \& Heckhausen, H. (2006). Eigenschaftstheorien der Motivation. In J. Heckhausen \& H. Heckhausen (Hrsg.), Motivation und Handeln (S. 43-72). Berlin: Springer.

Scheithauer, H. (2003). Aggressives Verhalten von Jungen und Mädchen. Göttingen: Hogrefe.

Schneider, H. J. (2007). Frauenkriminalität und Mädchendelinquenz. In H. J. Schneider (Hrsg.), Internationales Handbuch der Kriminologie. Band 1: Grundlagen der Kriminologie (S. 435-468). Berlin: De Gruyter.

Stadler, C., Janke, W. \& Schmeck, K. (2004). IVE: Inventar zur Erfassung von Impulsivität, Risikoverhalten und Empathie bei 9- bis 14-jährigen Kindern. Göttingen: Hogrefe.

Stams, J. G., Brugman, D., Dekovic, M., Rosmalen, L., Laan, P. \& Gibbs, J. C. (2006). The moral judgment of juvenile delinquents: A meta-analysis. Journal of Abnormal Child Psychology, 34, 697-713.

Svensson, R., Pauwels, L. \& Weerman, F. M. (2010). Does the effect of self-control on adolescent offending vary by level of morality? A test in three countries. Criminal Justice and Behavior, 37, 732- 743.

Walker, L. J. (2006). Gender and morality. In M. Killen \& J. Smetana (Hrsg.), Handbook of moral development. Mahwah New Jersey London: Lawrence Erlbaum Associates.

Walker, L. J. \& Pitts, R. C. (1998). Naturalistic conceptions of moral maturity. Developmental Psychology(34), 403-419.

Wikström, P.-O. H. \& Svensson, R. (2010). When does self-control matter? The interaction between morality and self-control in crime causation. European Journal of Criminology, 7, 395-410. 